\title{
Dynamical mass generation in pseudoquantum electrodynamics with Gross-Neveu interaction at finite temperature
}

\author{
Luis Fernández@ , $^{1, *}$ Reginaldo O. Corrêa, Jr.๑, ${ }^{2, \dagger}$ Van Sérgio Alves $\oplus^{1, \$}$ \\ Leandro O. Nascimento $\oplus^{3,8}$ and Francisco Peña $\odot^{4, \|}$ \\ ${ }^{1}$ Faculdade de Física, Universidade Federal do Pará, 66075-110 Belém, PA, Brazil \\ ${ }^{2}$ Centro de Ciências e Planetário Sebastião Sodré da Gama, Universidade do Estado do Pará, \\ Rodovia Augusto Montenegro km 03, 66640-000 Belém, PA, Brazil \\ ${ }^{3}$ Faculdade de Ciências Naturais, Universidade Federal do Pará, C.P. 68800-000 Breves, PA, Brazil \\ ${ }^{4}$ Departamento de Ciencias Físicas, Facultad de Ingeniería y Ciencias, Universidad de La Frontera, \\ Avenida Francisco Salazar 01145, Casilla 54-D, Temuco, Chile
}

(Received 20 September 2020; accepted 2 January 2021; published 20 January 2021)

\begin{abstract}
We describe the dynamical mass generation in pseudoquantum electrodynamics (PQED) coupled to the Gross-Neveu (GN) interaction, in $(2+1)$ dimensions, at both zero and finite temperatures. We start with a gapless model and show that, under particular conditions, a dynamically generated mass emerges. In order to do so, we use a truncated Schwinger-Dyson equation, at the large- $N$ approximation, in the imaginarytime formalism. In the instantaneous-exchange approximation (the static regime), we obtain two critical parameters, namely, the critical number of fermions $N_{c}(T)$ and the critical coupling constant $\alpha_{c}(T)$ as a function of temperature and of the finite cutoff $\Lambda$, which must be provided by experiments. In the dynamical regime, we find an analytical solution for the mass function $\Sigma(p, T)$ as well as a zero-external momentum solution for $p=0$. In the continuum theory $\Lambda=\infty$, where scale-invariance is respected, it is shown that the model has a dynamically generated mass for any value of the coupling constant $\alpha$. Furthermore, after calculating the effective potential for PQED, we prove that the dynamically generated mass is an energetically favorable solution in comparison to the massless phase. We compare our analytical results with numerical tests and a good agreement is found.
\end{abstract}

DOI: $10.1103 /$ PhysRevD.103.025018

\section{INTRODUCTION}

In the last decades, the interest in studying two-dimensional theories has been increased since the experimental realization of graphene [1,2] and other related materials, such as the transition metal dichalcogenide monolayers [3]. The main goal is to derive either new quantum phases of matter [4-7] or calculate renormalized parameters that change the electronic properties of these materials [8-13], opening possibilities for future technological applications (in particular spintronics [14], valleytronics [15], and electric-field tunning of energy bands $[16,17])$. Before this context, in the realm of high-energy physics, several works

\footnotetext{
*luis.aguilar@icen.ufpa.br †reginaldojunior@uepa.br *vansergi@ufpa.br

§lon@ufpa.br

"francisco.pena@ufrontera.cl
}

Published by the American Physical Society under the terms of the Creative Commons Attribution 4.0 International license. Further distribution of this work must maintain attribution to the author(s) and the published article's title, journal citation, and DOI. Funded by SCOAP ${ }^{3}$. discussed the possibility of dynamical mass generation for massless Dirac particles, yielding a phase transition to a new quantum state of matter in which the chiral symmetry is broken [18-21]. This is generated due to the electronic interactions in the plane and it may occur even at finite temperatures. Because electrons in these materials obey a Dirac-like equation, therefore, some authors have also considered the realization of dynamical mass generation in these two-dimensional systems [22-25].

The possibility of chiral symmetry breaking, i.e., dynamical mass generation in quantum electrodynamics (QED) has been discussed in several Refs. [18-21,26-28] for both $(2+1) \mathrm{D}$ and $(3+1) \mathrm{D}$ cases, just to cite a few. Furthermore, this symmetry-broken phase also has been shown to occur due to four-fermion interactions (such as Gross-Neveu and Thirring interactions) in Refs. [29-32]. In this case, the fermionic field exhibits a massive phase due to the spontaneous symmetry breaking, described by the so-called gap equation. This is usually calculated at the large- $N$ expansion, where $N$ is the number of fermion fields and the gap equation is calculated at order of $1 / N[29,32]$. The dynamical mass generation is a typical effect of the nonperturbative regime, hence, it is common the application of the Schwinger-Dyson equations (SDEs) 
for calculating the critical parameters that describe the phase transition.

The SDEs are an infinite set of coupled-integral equations, relating all of the interacting Green functions of the model $[19,20,33]$. Fortunately, within some approximative scheme, it is possible to find a truncated set of equations for calculating the desired Green functions, in particular, the electron-self energy that provides the chiral symmetry breaking. For pseudo-quantum electrodynamic (PQED) $[23,34]$, also called reduced quantum electrodynamics [35], the dynamical mass generation has been studied both at finite temperatures [36] and at zero temperature with the presence of the Gross-Neveu interaction [31]. Nevertheless, the effect of the Gross-Neveu interaction in PQED at finite temperatures has not been considered until now.

In this work, we describe the dynamical mass generation in PQED coupled to a Gross-Neveu interaction at finite temperature. We use the Matsubara formalism in order to include the effect of the thermal bath into the SchwingerDyson equation for the electron. This is dependent on both the gauge-field propagator and the auxiliary-field propagator, obtained after we use a Hubbard-Stratonovich transformation into the four-fermion interaction. These two bosonic propagators are calculated in order of $1 / N$, which is consistent with our assumption of strong-coupling limit. Thereafter, we use this result into the Schwinger-Dyson equation for the electron self-energy and calculate the full electron propagator in the dominant order of $1 / N$ in the nonperturbative limit. From this result, we conclude that a mass function is dynamically generated whether the number of fermions is less than a temperature-dependent critical parameter $N_{c}(T)$ for a finite cutoff $\Lambda$. In the continuum theory $\Lambda=\infty$, the mass generation occurs at any value of $\alpha$. At large temperatures, we find that $N_{c}(T) \ll 1$, hence, the dynamically generated mass vanishes and the system is in the gapless phase.

This paper is organized as follow. In Sec. II. we show our model and perform the large- $N$ approximation. In Sec. III. we write the truncated set of Schwinger-Dyson equations within the unquenched-rainbow approximation. In Sec. IV. we calculate the mass function in the static regime and obtain the critical parameters for the phase transition. In Sec. V. we use the zero-mode approximation in the dynamical regime for calculating the mass function. In Sec. VI. we summarize and discuss our main results. We also include four appendixes, where we give details about the angular integral, kernel expansion, the numerical results for the mass function, and show that the dynamically generated mass is the minimum of the effective potential.

\section{PSEUDOQUANTUM ELECTRODYNAMICS WITH GROSS-NEVEU INTERACTION}

We consider $N$ fermion fields constrained to the plane, whose interaction is described by pseudoquantum electrodynamics (PQED) [34]. Furthermore, we assume a contact interaction, given by the Gross-Neveu (GN) action [37]. Therefore, in the Euclidean space-time, the action of the model reads

$$
\begin{aligned}
\mathcal{L}= & \frac{1}{2} \frac{F_{\mu \nu} F^{\mu \nu}}{\sqrt{-\square}}+i \bar{\psi}_{a} \not \psi_{a}+e \bar{\psi}_{a} \gamma^{\mu} \psi_{a} A_{\mu} \\
& +-\frac{G_{0}}{2}\left(\bar{\psi}_{a} \psi_{a}\right)^{2}-\frac{\xi}{2} A_{\mu} \frac{\partial^{\mu} \partial^{\nu}}{\sqrt{-\square}} A_{\nu},
\end{aligned}
$$

where $F_{\mu \nu}$ is the field intensity tensor of $A_{\mu}$, which is our gauge field, and $\xi$ is the gauge-fixing parameter. On the other hand, $\psi_{a}$ is a four-component Dirac field, with the flavor index $a=1, \ldots, N$. The dimensionless coupling constant $e$ is the electric charge, $\gamma_{\mu}$ are the Dirac matrices in the four-rank representation, whose algebra is defined by $\left\{\gamma_{\mu}, \gamma_{\nu}\right\}=-2 \delta_{\mu \nu}$. The coupling constant $G_{0}$ describes the strength of the GN interaction and has unit of inverse of mass in the natural system of units $(\hbar=c=1)$.

In the perturbative regime at zero temperature, the model in Eq. (1) for massive fermions has been used to describe the renormalization of the band gap for $\mathrm{WSe}_{2}$ and $\mathrm{MoS}_{2}$ [13]. This result, nevertheless, requires a bare-mass term such as $m_{0} \bar{\psi}_{a} \psi_{a}$ which is renormalized at one-loop and provides a beta function for the mass in terms of the RG scale. From this result, one concludes that the renormalized mass is dependent on the electronic density and a good agreement with experimental data has been found in Ref. [13].

In the nonpertubative limit, the mass term is generated due to interactions even if we start with $m_{0} \rightarrow 0$ [31]. Here, we generalize this result by including a thermal bath of temperature $T$.

From Eq. (1), we find the gauge-field propagator, namely,

$$
\Delta_{0, \mu \nu}(p)=\frac{1}{2 \sqrt{p^{2}}}\left[\delta_{\mu \nu}-\left(1-\frac{1}{\xi}\right) \frac{p_{\mu} p_{\nu}}{p^{2}}\right]
$$

and the fermion propagator

$$
S_{0, F}(p)=-\frac{1}{\gamma^{\mu} p_{\mu}}
$$

Before we discuss the vertex interactions, let us apply the Hubbard-Stratonovich transformation, which converts the four-fermion interaction into a Yukawa-type interaction by including an auxiliary field $\varphi$ and the coupling constant $g=G_{0} N$. In this case, we replace the four-fermion interaction by the following scheme

$$
\mathcal{L}_{\mathrm{GN}} \rightarrow \mathcal{L}_{\mathrm{GN}}+\frac{1}{2 g}\left(\varphi-\frac{g}{\sqrt{N}} \bar{\psi}_{a} \psi_{a}\right)^{2},
$$

where $\mathcal{L}_{\mathrm{GN}}=-G_{0}\left(\bar{\psi}_{a} \psi_{a}\right)^{2} / 2$. Therefore, using Eq. (4) in Eq. (1), we find 


$$
\mathcal{L}_{\mathrm{GN}}=-\frac{\varphi}{\sqrt{N}} \bar{\psi}_{a} \psi_{a}+\frac{\varphi^{2}}{2 g} .
$$

Equation (5) must be supplemented by the motion equation of $\varphi$, given by

$$
\varphi=\frac{g}{\sqrt{N}} \bar{\psi}_{a} \psi_{a},
$$

which proves that the transformation does not change the dynamics of the model at classical level. Furthermore, the bare auxiliary-field propagator is

$$
\Delta_{0, \varphi}=\frac{1}{1 / g},
$$

and Eq. (1) reads

$$
\begin{aligned}
\mathcal{L}= & \frac{1}{2} \frac{F_{\mu \nu} F^{\mu \nu}}{\sqrt{-\square}}+i \bar{\psi}_{a} \not \psi_{a}+e \bar{\psi} \bar{\psi}_{a} \gamma^{\mu} \psi_{a} A_{\mu} \\
& -\frac{\varphi}{\sqrt{N}} \bar{\psi}_{a} \psi_{a}+\frac{\varphi^{2}}{2 g}-\frac{\xi}{2} A_{\mu} \frac{\partial^{\mu} \partial^{\nu}}{\sqrt{-\square}} A_{\nu} .
\end{aligned}
$$

The Yukawa-type vertex function is easily obtained from Eq. (8) and is given by $-1 / \sqrt{N}$. On the other hand, for summing the self-energies in the large- $N$ expansion for PQED, we shall replace $e^{2} \rightarrow \lambda / N$, where $\lambda$ is taken fixed at large $N$. This allow us to sum over all of the diagrammatic contributions in order of $1 / N$, which is an infinite sum, unlike the standard perturbation in $e$. Therefore, the PQED vertex reads $\sqrt{\lambda / N} \gamma_{\mu}$, describing the electromagnetic interaction.

\section{TRUNCATED SCHWINGER-DYSON EQUATION AT FINITE TEMPERATURES}

In this section we present the Schwinger-Dyson equations, obtained from Eq. (8), that describes the quantum corrections for the two-point functions. In principle, this is a very complicated set of integral equations for all of the full Green functions of the model. From now on, we assume the ladder approximation, also called rainbow approach [38], which consists of neglecting quantum corrections to the vertex functions. It is worthwhile to note that this may be corrected by the Ball-Chiu vertex [39] when one wishes to preserve the Ward-Takahashi identity.

Because we are interested in dynamical mass generation for the electrons, we need to find a closed solution for both gauge-field and auxiliary-field propagators. These shall be given by the large- $N$ approximation, thus the bosonic-field propagators are calculated in the unquenched approximation [40].

\section{A. Auxiliary field}

In Fig. 1, we show the diagrammatic representation of the Schwinger-Dyson equation for the auxiliary field.

Its analytical expression is given by [31]

$$
\Delta_{\varphi}^{-1}(p)=\Delta_{0, \varphi}^{-1}-\Pi(p),
$$

where $\Delta_{\varphi}^{-1}$ is the full propagator of $\varphi$ and $\Pi(p)$ is given by the fermionic loop, hence

$\Pi(p)=-\frac{1}{N} \operatorname{Tr} \int \frac{d^{3} k}{(2 \pi)^{3}} \rrbracket S_{F}(p-k) \Gamma(p, k) S_{F}(k)$,

where $S_{F}(p)$ is the full fermion propagator. In the lowest order of $1 / N$, we find that $\Pi(p)=-\sqrt{p^{2}} g_{0}$, with $g_{0}=$ $1 / 4$ [21]. Therefore, the full propagator of the auxiliary field is given by

$$
\Delta_{\varphi}(p)=\frac{1}{g_{0}} \frac{1}{\left(g g_{0}\right)^{-1}+\sqrt{p^{2}}} .
$$

\section{B. Gauge field}

In Fig. 2, we show the diagrammatic representation of the Schwinger-Dyson equation for the gauge-field propagator [31].

Its analytical expression is given by

$$
\Delta_{\mu \nu}^{-1}(p)=\Delta_{0, \mu \nu}^{-1}(p)-\Pi_{\mu \nu}(p),
$$

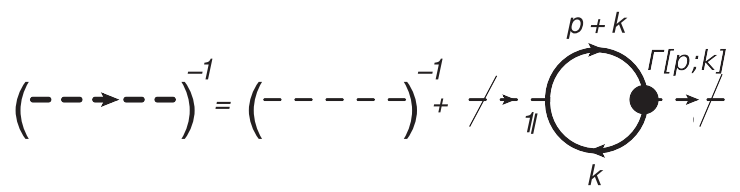

FIG. 1. The Schwinger-Dyson equation for the auxiliary field. The left-hand side is the inverse of the full propagator of the auxiliary field, the first term in the right-hand side corresponds to the bare auxiliary-field propagator, and the second term $\Pi(p)$ is the quantum correction. The function $\Gamma[p, k] \rightarrow \mathbb{1}$ corresponds to the approximated vertex.

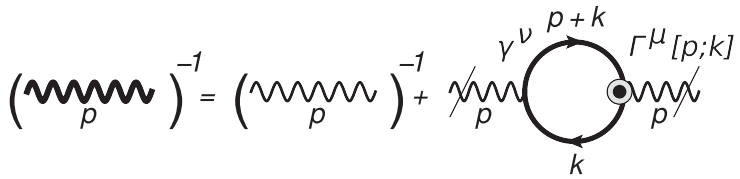

FIG. 2. The Schwinger-Dyson equation for the gauge-field propagator. The left-hand side is the inverse of the full propagator of the gauge field, the first in the right-hand side corresponds to the bare gauge-field propagator, and the second term $\Pi_{\mu \nu}(p)$ is the exact vacuum polarization tensor. The function $\Gamma^{\mu}[p, k] \rightarrow \gamma^{\mu}$ corresponds to the approximated vertex. 
where $\Delta_{\mu \nu}^{-1}$ is the full propagator and $\Pi_{\mu \nu}$ is the polarization tensor due to the electromagnetic interaction. This is given by

$$
\Pi_{\mu \nu}(p)=-\frac{\lambda}{N} \operatorname{Tr} \int \frac{d^{3} k}{(2 \pi)^{3}} \gamma_{\mu} S_{F}(p+k) \Gamma_{\nu}(p, k) S_{F}(k) .
$$

Similarly to the previous case, in the lowest order of $1 / N$, we find $\Pi_{\mu \nu}=\lambda \sqrt{p^{2}} / 8 P_{\mu \nu}$, where $P_{\mu \nu}=\delta_{\mu \nu}-p_{\mu} p_{\nu} / p^{2}$. Therefore, the full propagator reads

$$
\Delta_{\mu \nu}=\Delta_{0, \mu \alpha}\left[\delta_{\nu}^{\alpha}-\Pi^{\alpha \beta} \Delta_{0, \beta \nu}\right]^{-1} .
$$

Using Eqs. (2) and (13) in Eq. (14), we find

$$
\Delta_{\mu \nu}(p)=\frac{P_{\mu \nu}}{\sqrt{p^{2}}\left(2+\frac{\lambda}{8}\right)}
$$

in the Landau gauge, i.e., with $\xi=\infty$.

\section{Matter field}

In Fig. 3, we show the diagrammatic representation of the Schwinger-Dyson equation for the fermions field [31].

Its analytical expression is given by

$$
S_{F}^{-1}(p)=S_{0, F}^{-1}(p)-\Xi(p),
$$

where $S_{F}$ is the full fermion propagator and $\Xi$ is the electron self-energy, given by

$$
\Xi(p)=\Xi^{\lambda}(p)+\Xi^{g}(p),
$$

where $\Xi^{\lambda}$ is the PQED contribution and $\Xi^{g}$ is the GN contribution, namely,

$$
\Xi^{\lambda}(p)=\frac{\lambda}{N} \int \frac{d^{3} k}{(2 \pi)^{3}} \gamma^{\mu} S_{F}(k) \Gamma^{\nu}(k ; p) \Delta_{\mu \nu}(p-k),
$$

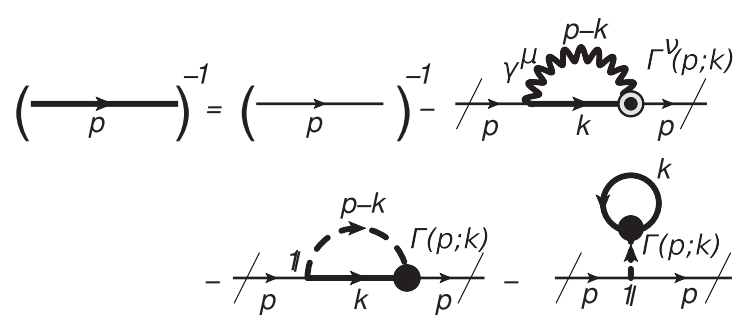

FIG. 3. The Schwinger-Dyson equation for the electron. The left-hand side is the inverse of the full propagator of the fermion field, the first term in the right-hand side corresponds to the bare Dirac propagator, and the other terms in the right-hand side are the electron self-energy $\Xi(p)$.

$$
\begin{aligned}
\Xi^{g}(p)= & \frac{1}{N} \int \frac{d^{3} k}{(2 \pi)^{3}} \mathbb{1} S_{F}(k) \Gamma(k ; p) \Delta_{\varphi}(p-k) \\
& +-\frac{1}{N} \int \frac{d^{3} k}{(2 \pi)^{3}} \rrbracket \operatorname{Tr}\left[S_{F}(k) \Gamma(k ; q=0) \Delta_{\varphi}(q=0)\right] .
\end{aligned}
$$

In order to find the full fermion propagator, it is convenient to decompose this Green function into its irreducible parts. Hence, we adopt the following ansatz

$$
S_{F}^{-1}(p)=-\gamma^{\mu} p_{\mu} A(p)+\Sigma(p)
$$

where $A(p)$ yields the wave function renormalization and $\Sigma(p)$ is called mass function. Note that $\Sigma(p) \neq 0$ implies a dynamical mass generation [21]. In order to obtain the mass function, we take the trace operation in both sides of Eq. (16). Thereafter, we substitute Eqs. (3), (18), (19), and (20), within the rainbow approximation, $\Gamma^{\nu}(p ; k) \rightarrow \gamma^{\nu}$ and $\Gamma(p ; k) \rightarrow \mathbb{1}$, we obtain

$$
\begin{aligned}
\Sigma(p)= & \frac{\lambda}{N} \int \frac{d^{3} k}{(2 \pi)^{3}} \frac{\delta^{\mu \nu} \Sigma(k)}{k^{2} A^{2}(k)+\Sigma^{2}(k)} \Delta_{\mu \nu}(p-k) \\
& +\frac{1}{N} \int \frac{d^{3} k}{(2 \pi)^{3}} \frac{\Sigma(k)}{k^{2} A^{2}(k)+\Sigma^{2}(k)} \Delta_{\varphi}(p-k) \\
& -\Delta_{\varphi}(0) \int \frac{d^{3} k}{(2 \pi)^{3}} \frac{\Sigma(k)}{k^{2} A^{2}(k)+\Sigma^{2}(k)} .
\end{aligned}
$$

On the other hand, for calculating the wave function renormalization, we multiply Eq. (16) by $\not p$ and, after calculating the trace operation, we obtain

$$
\begin{aligned}
A(p)= & 1-\frac{\lambda}{N p^{2}} \int \frac{d^{3} k}{(2 \pi)^{3}}\left(\delta^{\beta \mu} \delta^{\alpha \nu}-\delta^{\beta \alpha} \delta^{\mu \nu}\right. \\
& \left.+\delta^{\beta \nu} \delta^{\mu \alpha}\right) \frac{p_{\beta} k_{\alpha} A(k)}{k^{2} A^{2}(k)+\Sigma^{2}(k)} \Delta_{\mu \nu}(p-k) \\
& +\frac{1}{N p^{2}} \int \frac{d^{3} k}{(2 \pi)^{3}} \frac{\delta^{\beta \alpha} p_{\beta} k_{\alpha} A(k)}{k^{2} A^{2}(k)+\Sigma^{2}(k)} \Delta_{\varphi}(p-k) .
\end{aligned}
$$

It is clear that Eqs. (21) and (22) are a coupled set of equations for $\Sigma(p)$ and $A(p)$. However, in the dominant order $1 / N$, we may replace $A(p) \rightarrow 1$ in Eq. (21). This is also in agreement with the rainbow approximation.

Next, we use the Matsubara formalism for introducing temperature effects into Eq. (21). In the imaginary-time formalism, the main step is to change the time-component integrals into a sum over Matsubara frequencies [41], i.e., we must perform

$$
\int \frac{d k_{0}}{2 \pi} f\left(k_{0}, \mathbf{k}, \beta\right) \rightarrow \frac{1}{\beta} \sum_{n=-\infty}^{\infty} f(n, \mathbf{k}, \beta),
$$


with

$$
k_{\mu}=\left(k_{0}, \mathbf{k}\right) \rightarrow\left(\omega_{n}, \mathbf{k}\right)
$$

and

$$
\omega_{n}= \begin{cases}\frac{(2 n+1) \pi}{\beta} & \text { for fermions, } \\ \frac{2 n \pi}{\beta} & \text { for bosons }\end{cases}
$$

where $\omega_{n}$ are the Matsubara frequencies, $T=\beta^{-1}$ is the temperature of the thermal bath, and $n$ is the vibration mode related to each momentum component. Note that we are considering the Boltzmann constant as $k_{B}=1$, in the natural system of units. After including the thermal bath in Eq. (21), we have

$$
\begin{aligned}
\Sigma_{m}(\mathbf{p}, T)= & C_{1} \oint \frac{\Sigma_{n}(\mathbf{k}, T)}{(2 n+1)^{2} \pi^{2} T^{2}+\mathbf{k}^{2}+\Sigma_{n}^{2}(\mathbf{k}, T)} \\
& \times \frac{1}{\sqrt{4(n-m)^{2} \pi^{2} T^{2}+(\mathbf{p}-\mathbf{k})^{2}}} \\
& +C_{2} \oint \frac{\Sigma_{n}(\mathbf{k}, T)}{(2 n+1)^{2} \pi^{2} T^{2}+\mathbf{k}^{2}+\Sigma_{n}^{2}(\mathbf{k}, T)} \\
& \times \frac{1}{\left(g g_{0}\right)^{-1}+\sqrt{4(n-m)^{2} \pi^{2} T^{2}+(\mathbf{p}-\mathbf{k})^{2}}},
\end{aligned}
$$

where

$$
\begin{gathered}
\searrow \rightarrow T \sum_{n=-\infty}^{\infty} \int \frac{d^{2} k}{(2 \pi)^{2}}, \\
C_{1}=\frac{2 \lambda}{\left(2+\frac{\lambda}{8}\right) N},
\end{gathered}
$$

and

$$
C_{2}=\frac{1}{g_{0} N} .
$$

From now on, we assume that $\Sigma_{m}(\mathbf{p}, T)=\Sigma(\mathbf{p}, T)$ and, therefore, the mass function only depends on the dominant vibrational mode $m=0$. The standard procedure for calculating the mass function is to convert the integral equation into a differential equation for $\Sigma(\mathbf{p}, T)$. It turns out that there exist two main operations, namely, the sum over $n$ and the angular integral that must be performed before finding this result. We shall explore some approximations for these operations.

Before performing further approximations, let us show that the sum over the vibrational modes $n$ is convergent. The convergence of this sum for PQED at finite temperature has been shown in [36]. In Eq. (25), the sum over $n$ may be written as

$$
U=\sum_{n=-\infty}^{\infty} \frac{1}{(2 n+1)^{2}+A^{2}} \frac{1}{B+\sqrt{n^{2}+C^{2}}}
$$

where we conclude that $\left(A^{2}, C^{2}\right)>0$ and, because we consider $g>0[37,42]$, we also find that $B>0$.

Next, we apply the Cauchy integral test [43], where the sum is written as

$$
U=2 \sum_{n=1}^{\infty} u_{n}^{(+)}+u_{0},
$$

with $u_{n}^{(+)}=\left(u_{n}+u_{-n}\right) / 2$. The test imposes that whether $u(x)=u_{x}$ is positive, continuous, and decreasing in the interval $[1, \infty]$, hence, the integral over $u(x)$ is finite and, therefore, convergence is derived. This is exactly our case and one may easily conclude that

$$
\begin{gathered}
u(x)>0, \quad \forall x \mid x \in[1, \infty], \\
u^{\prime}(x)<0, \\
\lim _{x \rightarrow a} u(x)=u(a), \quad \forall a \mid a \in[1, \infty] .
\end{gathered}
$$

Therefore, Eq. (30) is a convergent series for all $n$.

\section{STATIC REGIME $p_{0}=0$}

The static regime, also called instantaneous-exchange approximation [44], consists of neglecting all of the timecomponents of the bosonic-field propagator in Eq. (21), with the consideration that the interaction vertex being only $\gamma^{0}$ in Eq. (18). This is usually realistic in cases where the electron velocity is much less than the light velocity. In these systems, it is possible to show that charged particles interacts through the Coulomb potential. This is given by the Fourier transform of the gauge-field propagator in Eq. (2) after we perform $p_{\mu}=\left(p_{0}, \mathbf{p}\right) \rightarrow(0, \mathbf{p})$, where we will use the following notation $(0, \mathbf{p}) \equiv(0, P)$. Next, let us consider both zero and finite temperature cases for calculating $\Sigma(P, T)$.

\section{A. Zero temperature case}

After considering the static regime with $T \rightarrow 0$ in Eq. (25), we find

$$
\begin{aligned}
\Sigma(P)= & \frac{C_{1}}{2} \int \frac{d^{3} k}{(2 \pi)^{3}} \frac{\Sigma(K)}{k_{0}^{2}+K^{2}+\Sigma^{2}(K)} \Delta(P-K) \\
& +C_{2} \int \frac{d^{3} k}{(2 \pi)^{3}} \frac{\Sigma(K)}{k_{0}^{2}+K^{2}+\Sigma^{2}(K)} \Delta_{\varphi}(P-K),
\end{aligned}
$$

where $d^{3} k=d k_{0} d^{2} K$, 


$$
\Delta_{\varphi}(P-K)=\frac{1}{\left(g g_{0}\right)^{-1}+\sqrt{(P-K)^{2}}},
$$

and

$$
\Delta(P-K)=\frac{1}{\sqrt{(P-K)^{2}}} .
$$

The integral over $k_{0}$ in Eq. (34) is easily solved and the angular integral may be solved by using the identity

$$
\int_{0}^{2 \pi} d \theta \Delta_{\varphi}(P-K)=\frac{4 K\left(x_{0}\right)}{\sqrt{(P-K)^{2}}}+O\left[\left(g g_{0}\right)^{-2}\right],
$$

where $K\left(x_{0}\right)$ is the complete elliptic integral of the first kind with $x_{0} \equiv-4 P K /(P-K)^{2}$. Note that the angular integral for the gauge-field term is very similar and that we will neglect the terms in order of $O\left[\left(g g_{0}\right)^{-2}\right]$. Replacing Eq. (37) in Eq. (34), we find

$\Sigma(P)=4\left(\frac{C_{1}}{2}+C_{2}\right) \int_{0}^{\Lambda} \frac{d K}{(2 \pi)^{2}} \frac{K \Sigma(K) \mathcal{K}(K, P)}{2 \sqrt{K^{2}+\Sigma^{2}(K)}}$,

where the kernel $\mathcal{K}(K, P)$ reads

$$
\mathcal{K}(K, P)=\frac{K\left(x_{0}\right)}{\sqrt{(P-K)^{2}}} .
$$

Next, we approximate the kernel for its lowest order terms, namely,

$$
\mathcal{K}(K, P) \approx \frac{\pi}{2 P} \Theta(P-K)+\frac{\pi}{2 K} \Theta(K-P) .
$$

Note that $\Theta(P-K)$ is the standard step function. Using Eq. (40) in Eq. (38), we have

$$
\begin{aligned}
\Sigma(P)= & \frac{\left(C_{1} / 2+C_{2}\right)}{4 \pi}\left[\int_{0}^{P} K d K \frac{\Sigma(K)}{P \sqrt{K^{2}+\Sigma^{2}(K)}}\right. \\
& \left.+\int_{P}^{\Lambda} d K \frac{\Sigma(K)}{\sqrt{K^{2}+\Sigma^{2}(K)}}\right] .
\end{aligned}
$$

On the other hand, by taking derivatives in respect to $P$ in both sides of Eq. (41), we obtain a differential equation for the mass function, given by

$$
P^{2} \Sigma^{\prime \prime}(P)+2 P \Sigma^{\prime}(P)+\frac{N_{c}}{4 N} \frac{P \Sigma(P)}{\sqrt{P^{2}+\Sigma^{2}(P)}}=0,
$$

where the critical number of fermions is given by

$$
N_{c}=\frac{1}{\pi}\left(\frac{C_{1}}{2}+C_{2}\right)=\frac{\lambda}{\pi\left(2+\frac{\lambda}{8}\right)}+\frac{4}{\pi} .
$$

Equation (42) is also supplemented by two equations, namely,

$$
\lim _{P \rightarrow 0} P^{2} \Sigma^{\prime}(P)=0,
$$

which provides the infrared behavior (IR) and

$$
\lim _{P \rightarrow \Lambda}\left[P \Sigma^{\prime}(P)+\Sigma(P)\right]=0,
$$

which shows that the mass function is expected to vanish at large $P$, which is our ultraviolet (UV) condition.

Using the approximation $P / \sqrt{P^{2}+\Sigma^{2}(P)} \approx 1$ in Eq. (42), which holds for large-external momentum, we find the analytical solution

$$
\Sigma(P)=A_{1} P^{\gamma}+A_{2} P^{\gamma^{*}},
$$

where $\gamma=-1 / 2+i / 2 \sqrt{N_{c} / N-1}$, and $\left(A_{1}, A_{2}\right)$ are arbitrary constants.

The analytical solution in Eq. (46) must be in agreement with both IR and UV conditions in Eq. (44) and Eq. (45), respectively. This is a standard step in order to calculate the critical behavior of $\Sigma(P)$. First, we may consider the limit where $\Lambda=\infty$, which represents the continuum theory. Thereafter, it is easy to show that $\Sigma(P)$ obeys both IR and UV conditions for any value of $N$. Therefore, because $N_{c}$ in Eq. (43) is dependent on $\lambda=4 \pi \alpha N$, we also conclude that the dynamical mass generation occurs for any value of $\alpha$ (or $\lambda$ ). This continuum limit has been discussed for PQED (in the dynamical limit) in Ref. [23] and for $\mathrm{QED}_{4}$ it has been reviewed in Ref. [19], where the same conclusion is derived.

Next, we assume that the system has a finite cutoff $\Lambda \propto 1 / a$, where $a \approx 1 \AA$ is the lattice parameter. This is motivated by an effective description of electrons in condensed matter physics $[2-4,7,12]$. In this case, the IR condition in Eq. (44) is also respected for any value of $N$. On the other hand, the analysis of the UV condition in Eq. (45) is more subtle. Indeed, for the sake of simplicity, let us define $\gamma=-1 / 2+i R / 2$ with $R \equiv \sqrt{N_{c} / N-1}$ in Eq. (46). Clearly, we have two cases, i.e., (i) where $R$ is imaginary and $N>N_{c}$ and (ii) where $R$ is real and $N<N_{c}$. In the first case, after using Eq. (46) in Eq. (45), it follows that $\Sigma(P) \neq 0$ does not obey the UV condition and, therefore, we only find the trivial solution $\Sigma(P)=0$.

For the second case, $R$ is real and $\Sigma(P)$ may be written as

$$
\Sigma(P)=\frac{\bar{A}}{\sqrt{P}} \sin \left[R\left(\ln \frac{P}{M_{\Lambda}}+\phi\right)\right],
$$

where $\bar{A}=\sqrt{C_{1}^{2}-C_{2}^{2}}, \quad C_{1}=A_{1}+A_{2}, \quad C_{2}=A_{2}-A_{1}$, $R \phi=\tan ^{-1}\left(-i C_{1} / C_{2}\right)$, and $M_{\Lambda}$ is a massive parameter. Furthermore, using Eq. (47) in Eq. (45), we find that 
this massive parameter obeys a Miransky-scaling law, namely,

$$
M_{\Lambda}=\Lambda e^{2+R} e^{-2 n \pi / R}
$$

where $n$ is an integer. The typical values of $M_{\Lambda}$ ensures that $\Sigma(P)$ obeys the UV condition and, therefore, we find a massive phase only for $N<N_{c}$. Interesting, the numerical results in Ref. [23] indicates that $\Sigma(0)$ (the maximum value of $\Sigma(P)$ occurs at $P=0$ ) also obeys a Miransky-scaling law, given by $\Sigma(0) \propto \Lambda e^{-1 / \sqrt{N-N_{c}}}$. This shows that $\Sigma(0) \rightarrow 0$ for $N \rightarrow N_{c}$, which confirms the interpretation of $N=N_{c}$ as a critical point. A similar result also holds for quantum electrodynamics in $(2+1)$ dimensions [18,20,21] and $(3+1)$ dimensions [19]. From now on, let us assume that $\Lambda$ is finite.

Because $N_{c}$ is a function of $\alpha$ in Eq. (43), we may define a critical coupling constant $\alpha_{c}$ (or $\lambda_{c}$ ) through the critical point $R=\sqrt{N_{c} / N-1} \rightarrow 0$, which occurs for $N_{c}\left(\lambda_{c}\right)=N$ with $\lambda_{c}=4 \pi \alpha_{c} N$. Therefore, it follows that

$$
\alpha_{c}=\frac{\frac{16}{N \pi}-4}{N \pi-12}
$$

which, in terms of the fine-structure constant, implies that the mass generation occurs for $\alpha>\alpha_{c}$ and $N<N_{c}(\alpha)$. Finally, from Eq. (43), we conclude that $N_{c}(\lambda=0) \leq N_{c} \leq$ $N_{c}(\lambda=\infty)$, where $N_{c}(\lambda=0)=4 / \pi \approx 1.27$ and $N_{c}(\lambda=\infty)=$ $12 / \pi \approx 3.82$. This result may be compared with the critical point calculated in the dynamical limit in Ref. [31]. In this case, the value of the critical number is $0.81 \leq N_{c}^{\text {dyn. }} \leq 4.05$. These results suggest that, in the strong-coupling limit, it is more favorable to find a massive phase in the dynamical limit, where $p_{0} \neq 0$ in the gaugefield propagator and the vertex interaction $e \gamma^{\mu}$ is taken into account.

\section{B. Finite temperature}

In order to describe temperature effects, we include the Matsubara frequencies in Eq. (34). Thereafter, we solve the angular integral using Eq. (37) while for the Matsubara sum we use the identity

$$
T \sum_{n} \frac{1}{\omega_{n}^{2}+\epsilon_{k}^{2}}=\frac{1}{2 \epsilon_{k}}\left[1-2 n_{F}\left(\epsilon_{k}\right)\right],
$$

where $\epsilon_{K} \equiv \sqrt{K^{2}+\Sigma^{2}(K)}$ and

$$
n_{F}\left(\epsilon_{K}\right)=\frac{1}{e^{\beta \epsilon_{K}}+1}
$$

is the Fermi-Dirac distribution. After using these properties, we find
$\Sigma(P, T)=4\left(\frac{C_{1}}{2}+C_{2}\right) \int_{0}^{\Lambda} d K \frac{K}{(2 \pi)^{2}} \frac{\Sigma(K) \mathcal{K}_{\beta}(K, P)}{2 \sqrt{K^{2}+\Sigma^{2}(K)}}$,

where the temperature-dependent kernel $\mathcal{K}_{\beta}(K, P)$ reads

$$
\mathcal{K}_{\beta}(K, P)=\frac{K\left(x_{0}\right)}{\sqrt{(P-K)^{2}}}\left[1-2 n_{F}\left(\epsilon_{K}\right)\right] .
$$

Next, following the same steps as in the previous section, we find the differential equation for the mass function, namely,

$P^{2} \Sigma^{\prime \prime}(P)+2 P \Sigma^{\prime}(P)+\frac{N_{c} h_{\beta}(P)}{4 N} \frac{P \Sigma(P)}{\sqrt{P^{2}+\Sigma^{2}(P)}}=0$,

where

$$
h_{\beta}(P)=\left[1-2 n_{F}\left(\epsilon_{P}\right)\right] .
$$

The linearized version of Eq. (54) may be obtained at largeexternal momentum and by doing $P \rightarrow \Lambda$ in $h_{\beta}(P)$. This allow us to obtain a temperature-dependent critical number $N_{c}(T)$, given by

$$
N_{c}(T)=N_{c}(0)\left[1-\frac{2}{e^{\Lambda / T}+1}\right],
$$

where $N_{c}(0) \equiv N_{c}=\left(C_{1} / 2+C_{2}\right) / \pi$ is given by Eq. (43) in terms of $\lambda$. On the other hand, for large- $T$, we obtain $N_{c}(T) \rightarrow 0$, which implies that there exist no dynamical mass generation for any $\lambda$.

In the large-external momentum, the solution of Eq. (54) is given by Eq. (46), after we replace $N_{c} \rightarrow N_{c}(T)$. Hence, we obtain a temperature-dependent exponent $\gamma(T)=$ $-1 / 2+i / 2 \sqrt{N_{c}(T) / N-1}$. Therefore, we find

$$
\Sigma(P, T)=A_{1}(T) P^{\gamma(T)}+A_{2}(T) P^{\gamma^{*}(T)},
$$

where the arbitrary constants $\left(A_{1}, A_{2}\right)$ may be dependent on the temperature. Because both the IR/UV conditions and the momentum dependence of the solution $\Sigma(P, T)$ are the same as in the zero-temperature case, it follows the same critical behavior discussed before. In particular, for finite $\Lambda$, the dynamical mass generation only occurs for $N<N_{c}(T)$. Furthermore, similar to the zero-temperature case, this critical point also may be described in terms of a temperature-dependent critical constant $\alpha_{c}(T)$, given by

$$
\alpha_{c}(T)=-\frac{4\left[4\left(1-e^{\Lambda / T}\right)+N \pi\left(1+e^{\frac{\Lambda}{T}}\right)\right]}{N \pi\left[12\left(1-e^{\Lambda / T}\right)+N \pi\left(1+e^{\frac{\Lambda}{T}}\right)\right]} .
$$

Note that for $T \rightarrow 0$, Eq. (58) yields Eq. (49), as expected. In terms of $\alpha$, the dynamical mass generation only occurs 


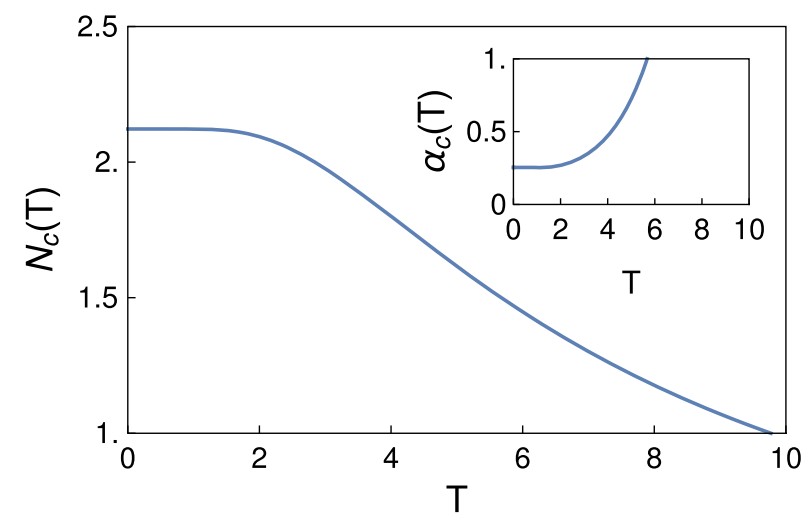

FIG. 4. The critical parameters for the dynamical mass generation. We plot both Eq. (56) (the common line) and Eq. (58) (the inset) with $\Lambda=10$ (units of energy). For the common line we also use $\lambda=8.0$, while for the inset we use $N=2$. Note that for small enough temperatures, the critical coupling constants remain almost unchanged.

when $\alpha>\alpha_{c}(T)$ and $N<N_{c}(T)$. In Fig. 4, we plot both $N_{c}(T)$ and $\alpha_{c}(T)$ and show that whether the temperature increases, hence, the system quickly goes to a gapless phase.

We also may find numerical results (see Appendix A for more details) of the integral equation for $\Sigma(P, T)$ in Eq. (52). In Fig. 5, we plot these results and compare this with our analytical solution given by Eq. (57).

\section{DYNAMICAL REGIME $p_{0} \neq 0$}

In this section we recover the retardation effects by assuming $p_{0} \neq 0$ in Eq. (21). For $T=0$, this regime has been discussed in several contexts.

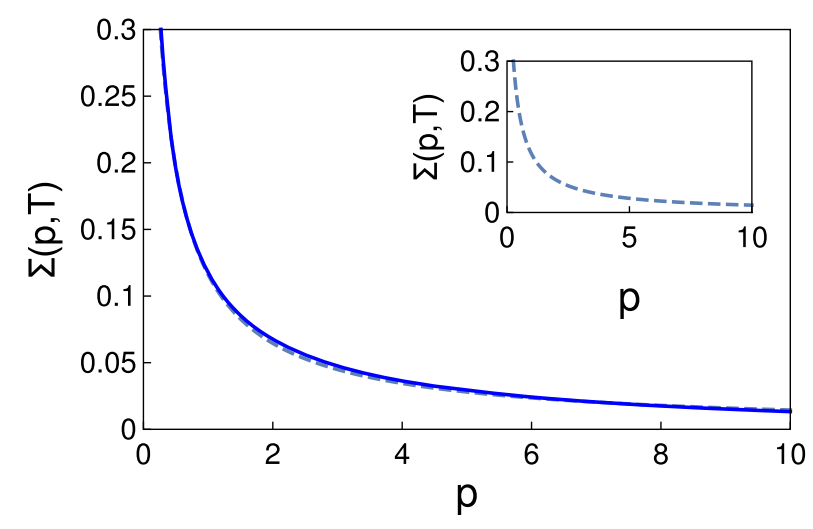

FIG. 5. The analytical and numerical solutions for $\Sigma(P, T)$. The dashed line is the numerical solution of the integral equation given by Eq. (52) with $N=2$. The common line is the analytical solution given by Eq. (57) with $N=2$ and $A_{1}(T)=A_{2}(T)^{*}=$ $0.06+0.13 i$. We have used $\Lambda=10$ (units of energy), $\lambda=8.0$, and $T=0.1$ (units of $\Lambda$ ) for all of the curves. We include an inset in order to facilitate the visualization of the numerical solution. Note that the analytical solution is in good agreement with the numerical results.
After a simple changing of variables, the angular integral of the PQED sector (see details in Appendix B) reads

$$
\mathcal{I}_{n}^{\mathrm{PQED}}(p, k ; T)=\frac{4}{w_{p, k}^{n}} K\left[x_{n}\right]
$$

where,

$$
w_{p, k}^{n}=\sqrt{4 n^{2} \pi^{2} T^{2}+(p-k)^{2}}
$$

and

$$
x_{n}=-\frac{4 p k}{4 n^{2} \pi^{2} T^{2}+(p-k)^{2}} .
$$

Similarly, for the GN sector, we find

$$
\mathcal{I}_{n}^{\mathrm{GN}}(p, k, T)=\frac{4}{w_{p, k}^{n}}\left[K\left(x_{n}\right)-\frac{\Pi\left(y_{n}, x_{n}\right)}{1-g^{2} g_{0}^{2}\left(w_{p, k}^{n}\right)^{2}}\right]
$$

with

$$
y_{n}=-\frac{4 p k}{4 n^{2} \pi^{2} T^{2}+(p-k)^{2}-\frac{1}{g^{2} g_{0}^{2}}},
$$

where $K\left(x_{n}\right)$ and $\Pi\left(y_{n}, x_{n}\right)$ are the elliptic integral of the first and third kind, respectively. In particular, note that for $g=0$, we have that $\Pi\left(0, x_{n}\right)=K\left(x_{n}\right)$, hence, the kernel of the GN sector vanishes, as expected. The integral representation for these functions are

$$
K\left(x_{n}\right)=\int_{0}^{\pi / 2} d \theta \frac{1}{\sqrt{1-x_{n} \sin ^{2}(\theta)}}
$$

and

$\Pi\left(y_{n}, x_{n}\right)=\int_{0}^{\pi / 2} d \theta \frac{1}{\left[1-y_{n} \sin ^{2}(\theta)\right] \sqrt{1-x_{n} \sin ^{2}(\theta)}}$.

The resulting kernel, after using the angular integral, is a very complicated function of $n$. This preclude us of solving the whole Matsubara sum, as we have did in the static regime. Therefore, we shall investigate the lowest order contribution, provided by the zero-mode $n=0$ approximation.

\section{ZERO-MODE APPROXIMATION}

In this section we consider the fundamental vibrational mode $n=0$ in Eq. (25). Using Eqs. (64) and (65), we find 


$$
\begin{aligned}
\Sigma(p, T)= & \frac{C_{1}}{\pi^{2}} \int_{0}^{\infty} d k \frac{k K\left(x_{0}\right)}{\|p-k\|} \frac{T \Sigma(k, T)}{\pi^{2} T^{2}+k^{2}+\Sigma^{2}(k, T)} \\
& +\frac{C_{2}}{\pi^{2}} \int_{0}^{\infty} d k \frac{k}{\|p-k\|} \frac{T \Sigma(k, T)}{\pi^{2} T^{2}+k^{2}+\Sigma^{2}(k, T)} \\
& \times\left[K\left(x_{0}\right)-\frac{\Pi\left(y_{0}, x_{0}\right)}{1-g^{2} g_{0}^{2}(p-k)^{2}}\right]
\end{aligned}
$$

For calculating an analytical solution of Eq. (66), we write the elliptic integral as the hypergeometrics Appell $F_{1}$. Because $F_{1}$ has an expansion in its parameters, we may find a simplified version of the kernel (see Appendix C for more details). Hence, Eq. (66) yields

$$
\begin{aligned}
\Sigma(p)= & \frac{C_{1} T}{2 \pi} \int_{0}^{\Lambda} d k f(k)\left[\frac{\Theta(p-k)}{p}+\frac{\Theta(k-p)}{k}\right] \\
& +\frac{C_{2} T}{2 \pi} \int_{0}^{\Lambda} d k f(k)\left[\frac{\Theta(p-k)}{p}+\frac{\Theta(k-p) k}{k^{2}-\frac{1}{\left(g g_{0}\right)^{2}}}\right],
\end{aligned}
$$

where $f(k) \equiv k \Sigma(k) /\left[(\pi T)^{2}+k^{2}+\Sigma^{2}(k)\right]$. By taking derivatives in respect to $p$ in both sides of Eq. (67), we find

$\Sigma^{\prime}(p)+\frac{b \Sigma(p)}{(\pi T)^{2}+p^{2}+\Sigma^{2}(p)}=-\frac{a}{p^{2}} \int_{0}^{p} d k f(k)$,

with $a=\left(C_{1}+C_{2}\right) T / 2 \pi$ and $b=C_{2} T / 2 \pi$, both constants with dimension of mass. Next, we neglect the nonlinear terms by using $(\pi T)^{2}+p^{2}+\Sigma^{2}(p) \approx(\pi T)^{2}+p^{2}$, which is expected to be reasonable at large $p[36,45]$. After deriving Eq. (68) in respect to $p$, we obtain the differential equation

$$
\begin{aligned}
& p^{3} \Sigma^{\prime \prime}(p)+\left[2 p^{2}+\frac{p^{3} b}{(\pi T)^{2}+p^{2}}\right] \Sigma^{\prime}(p) \\
& \quad+\left[\frac{2 p^{2} b}{(\pi T)^{2}+p^{2}}-\frac{2 p^{4} b}{\left((\pi T)^{2}+p^{2}\right)^{2}}+a\right] \Sigma(p)=0,
\end{aligned}
$$

which is supplemented by two conditions for the infrared and ultraviolet regimes. These are given by

$$
\lim _{p \rightarrow \Lambda} \Sigma(p)=0
$$

and

$$
\lim _{p \rightarrow 0} p^{2}\left[\frac{d \Sigma(p)}{d p}+\frac{b \Sigma(p)}{(\pi T)^{2}+p^{2}}\right]=0
$$

Unfortunately, Eq. (69) has not an analytical solution for arbitrary values of the set $(p, T)$ of variables. Nevertheless, we may consider a possible linearized version for large external momentum.

\section{A. Large-external-momentum approximation}

In this section we investigate the linearized version of Eq. (69) by assuming $p \gg T, \Sigma(p)$. In this case, the behavior of the generated mass is given by

$$
p^{3} \Sigma^{\prime \prime}(p)+\left[2 p^{2}+p b\right] \Sigma^{\prime}(p)+a \Sigma(p)=0
$$

which yields

$\Sigma(p)=A_{1} \frac{b_{1} F_{1}\left[1-\frac{a}{b}, 2, \frac{b}{p}\right]}{p}+A_{2} G_{12}^{20}\left[-\frac{b}{p} \mid \begin{array}{c}\frac{a+b}{b} \\ 0,1\end{array}\right]$

where ${ }_{1} F_{1}$ is the confluent hypergeometric function and $\mathrm{G}$ is the Meijer G-function [46]. The sign of this Meijer G-function changes for different values of the external momentum. Because we have not observed such behavior in the numerical results of Eq. (66), from now on, we shall take $A_{2}=0$ for the sake of agreement with the integral equation. In Fig. 6, we plot our analytical solution in Eq. (73) and compare this with the numerical results of the integral equation in Eq. (66).

\section{B. Zero-external-momentum approximation}

In this section, we consider the limit $\Sigma(p=0, T)=$ $m(T)$ in Eq. (25), using the zero-frequency mode. In this case, the angular integral provides a constant factor of $2 \pi$. Hence, we find

$$
\begin{aligned}
1= & \frac{1}{4 \pi} \int_{0}^{\Lambda} d k \frac{1}{\pi^{2} T^{2}+k^{2}+m^{2}(T)} \\
& \times\left\{C_{1} T+\frac{C_{2} T k}{\left(g g_{0}\right)^{-1}+k}\right\} .
\end{aligned}
$$

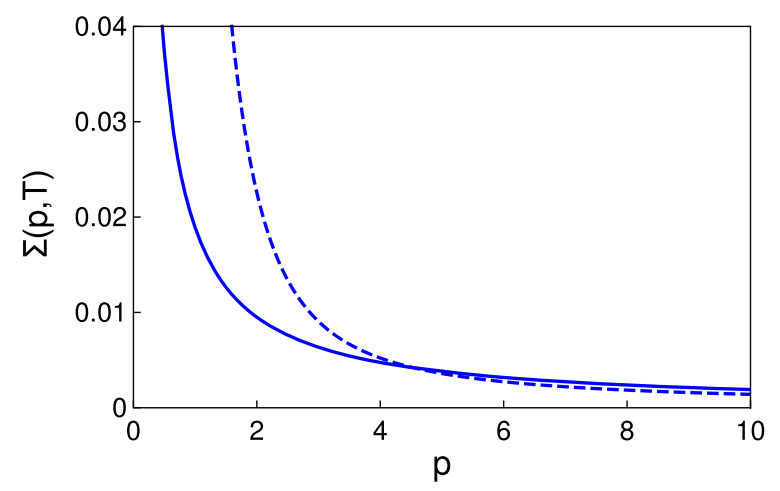

FIG. 6. The analytical and numerical solutions for $\Sigma(p, T)$ in the zero-mode approximation. The dashed line is the numerical solution of the integral equation given by Eq. (66) with $N=2$. The common line is the analytical solution given by Eq. (73) with $N=2$ and $A_{1}(T)=0.6$. We have used $\Lambda=10$ (units of energy), $\lambda=3.0$, and $T=0.1$ (units of $\Lambda$ ) for all of the curves. Note that the analytical solution is in good agreement with the numerical results only at large- $p$ limit. 
After calculating the integral over $k$, we have

$$
\begin{aligned}
1= & \frac{C_{1} T}{2 \pi} \frac{\arctan \left(\frac{\Lambda}{\sqrt{\pi^{2} T^{2}+m^{2}(T)}}\right)}{\sqrt{m^{2}(T)+\pi^{2} T^{2}}} \\
& +\frac{C_{2} T}{2 \pi}\left\{\frac{\sqrt{\pi^{2} T^{2}+m^{2}(T)} \arctan \left(\frac{\Lambda}{\sqrt{\pi^{2} T^{2}+m^{2}(T)}}\right)}{\left[\pi^{2} T^{2}+m^{2}(T)+\left(\frac{1}{g g_{0}}\right)^{2}\right]}\right. \\
& +\frac{g g_{0} \ln \left[\frac{\pi^{2} T^{2}+m^{2}(T)+\Lambda^{2}}{2\left[\left(g g_{0}\right)^{2}\left(\pi^{2} T^{2}+m^{2}(T)\right)+1\right]}\right\} .}{} .
\end{aligned}
$$

Equation (75) is our gap equation for the dynamical mass as a function of temperature and the coupling constants. Similarly to the BCS model, we define the critical temperature as the point $T=T_{c}$ in which $m$ vanishes. Here, nevertheless, we define that $C_{1} T$ and $C_{2} T$ are fixed in order to be in agreement with the fact that $m \neq 0$ for $T=0$.

In Fig. 7, we plot the numerical solutions of $m(T)$ for both $g=0$ and $g=1.0$, which proves that the GN interaction increases the gapped phase. Furthermore, in Fig. 8, we show that the GN interaction increases the critical temperature, as expected.

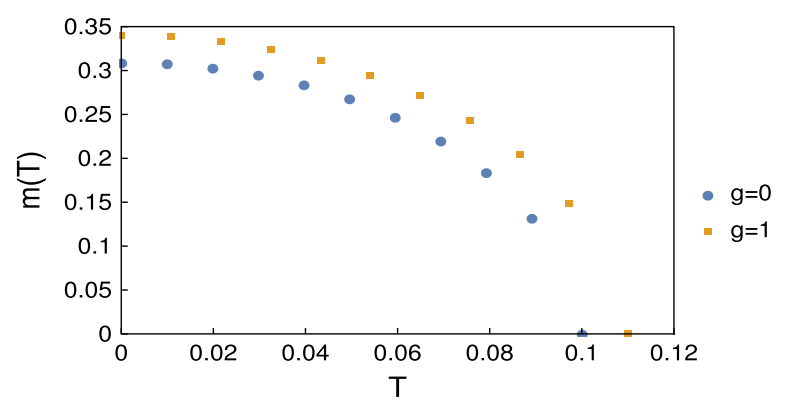

FIG. 7. The numerical result of the mass function given by Eq. (75). We use $\Lambda=10, N=2$ and $\lambda=3$ in the blue points with $g=0$, and in the square point $g=1$. Where, we see the augment of the critical temperature due at the GN-coupling.

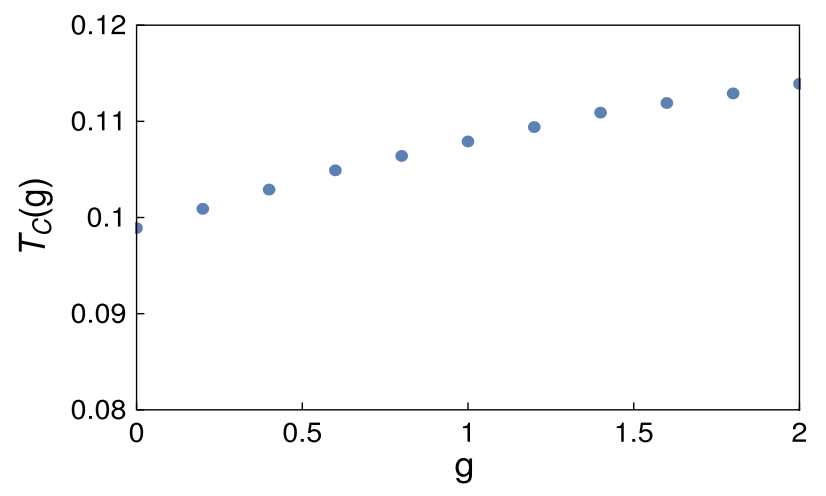

FIG. 8. Behavior of critical temperature for different values of the GN coupling constant given by Eq. (75) numerically treated. Where we use $\Lambda=10, N=2$ and $\lambda=3$.
We have shown that a dynamical mass is generated and, therefore, it is expected to observe a massive phase. Nevertheless, one also needs to prove that this is indeed a favorable phase in comparison to the symmetric phase. It turns out that PQED has the same vertex interaction as in $\mathrm{QED}_{3}$, namely, $e \gamma^{\mu}$, which implies the same SchwingerDyson equations, except by the different gauge-field propagator. Therefore, we can safely follow the same steps in Ref. [47] for $\mathrm{QED}_{3}$ and conclude that the nontrivial solution is the minimum of the effective potential (See Appendix D for more details).

\section{SUMMARY AND OUTLOOK}

In this work, we investigate the influence of temperature on the dynamical mass generation for fermions in PQED with a Gross-Neveu interaction. In order to do so, we used the Schwinger-Dyson equation for the electron in the dominant order $1 / N$, neglecting quantum corrections to the vertices at finite temperature. In the static regime, we were able to solve the whole Matsubara sum. Thereafter, we calculated the critical coupling constant $\alpha_{c}$ and the critical numbers of fermions $N_{c}$ as a function of $T$ and the cutoff $\Lambda$. From these results, we concluded that the system goes to a gapless phase whether we increase the ratio $T / \Lambda$. In the continuum theory $\Lambda=\infty$, where scale-invariance is respected, the dynamical mass generation occurs for any value of $\alpha$ [23]. Furthermore, we obtained an analytical solution for the mass function $\Sigma(p)$, which is in good agreement with our numerical results.

In the dynamical regime, we used the zero-mode approximation in the sum over the Matsubara frequencies, which allowed us to calculate the analytical solution $\Sigma(p)$. This function agrees with our numerical results for largeexternal momentum, while for small-external momentum some deviations have been found. Moreover, we found a gap equation that provides the value of the mass function in the zero-external momentum approximation, i.e., $\Sigma(p=0, T) \rightarrow m(T)$. In this case, the numerical tests show that the critical temperature increases as we increase the strength of the fermionic self-interaction.

Several generalizations of this work may be performed. For instance, one would investigate the main effects of vertices quantum corrections into the critical parameters for the mass generation. In particular, it would be relevant to find a way to increase the gapped phase for finite temperature. In this phase, the competition between $\alpha$ and $g$ yields a tunable mass which may be relevant for applications in two-dimensional materials [13].

\section{ACKNOWLEDGMENTS}

L.F. is partially supported by Coordenação de Aperfeiçoamento de Pessoal de Nível Superior Brasil (CAPES), finance code 001. V. S. A. and L. O. N. are partially supported by Conselho Nacional de Desenvolvimento 
Científico e Tecnológico (CNPq) and by CAPES/NUFFIC, finance code 0112. F. P. acknowledge the financial support from Dirección De Investigación De La Universidad De La Frontera Grant No. DI20-0005 of the Dirección de Investigación y Desarrollo, Universidad de La Frontera.

\section{APPENDIX A: THE NUMERICAL RESULTS}

In this Appendix we briefly review the main steps in order to find our numerical results. For the sake of simplicity, note that Eq. (25) is a kind of Fredholm integral equation of first kind, defined by

$$
Z(x)=\int_{a}^{b} d y \mathcal{K}(x, y, Z(y)) Z(y), \quad(a \leq x \leq b)
$$

where $\mathcal{K}(x, y, Z(y))$ is the kernel of the integral equation and $Z(x)$ is an unknown function. Throughout this work we convert this into a differential equation, allowing us to find analytical solutions for each approximation, namely, Eq. (57) in the static approximation and Eq. (73) in the zero-mode approximation. These solutions, nevertheless, are not expected to well describe the behavior of the mass function for small external momentum.

For properly describing the behavior of $\Sigma(p)$ for any $p$, we may find numerical results of both Eq. (52) and Eq. (67). It is easy to conclude that these equations are essentially a kind of Fredholm integral equation whether we adjust the parameters, variables, and kernel. Next, let us summarize our main steps. First, we use the trapezoidal quadrature rule for calculating the integral over the kernel, hence,

$$
\begin{array}{rl}
\int_{a}^{b} & d y \mathcal{K}(x, y, Z) Z(y) \\
\rightarrow & \frac{h}{2} \sum_{i=1}^{M-1}\left[\mathcal{K}\left(y_{i}, x, Z_{i}\right) Z_{i}+\mathcal{K}\left(y_{i+1}, x, Z_{i+1}\right) Z_{i+1}\right]
\end{array}
$$

where $h$ is the size of each interval and $y_{i}$ is the iterative variable (plays the role of loop momentum $k$ ). Setting $M=$ 300 as the numbers intervals, hence, $h=(a-b) /(M-1)$, with $a=10$ (plays the role of the $\Lambda$ cutoff), and $b=10^{-3}$ in order to avoid divergences. Furthermore, note that $10^{-3} \leq\left(x, y_{i}\right) \leq 10$. Using Eq. (A2) in Eq. (A1), we obtain a set of algebraic equations for all of $Z_{i}$ from $x=b$ to $x=a$. Finally, after solving this set of equations, we find Figs. 5 and 6.

\section{APPENDIX B: ANGULAR INTEGRAL}

The mass function given by Eq. (25) has two main contributions, generated by PQED and GN interactions. In the polar system of coordinates, it yields

$$
\begin{aligned}
\Sigma(p)= & C_{1} T \sum_{n=-\infty}^{\infty} \int_{0}^{\infty} \frac{d k}{(2 \pi)^{2}} \frac{k \Sigma(k)}{(2 n+1)^{2} \pi^{2} T^{2}+k^{2}+\Sigma^{2}(k)} \\
& \times \int_{0}^{2 \pi} d \theta \frac{1}{\sqrt{4 n^{2} \pi^{2} T^{2}+p^{2}+k^{2}-2 k p \cos (\theta)}} \\
& +C_{2} T \sum_{n=-\infty}^{\infty} \int_{0}^{\infty} \frac{d k}{(2 \pi)^{2}} \frac{k \Sigma(k)}{(2 n+1)^{2} \pi^{2} T^{2}+k^{2}+\Sigma^{2}(k)} \\
& \times \int_{0}^{2 \pi} d \theta \frac{1}{\frac{1}{g g_{0}}+\sqrt{4 n^{2} \pi^{2} T^{2}+p^{2}+k^{2}-2 k p \cos (\theta)}} .
\end{aligned}
$$

After we use the identity $\cos (\theta)=1-2 \sin ^{2}(\theta / 2)$ and a change of variable $(\theta \rightarrow 2 \theta)$ in the angle, it is possible to write the PQED angular integral as

$\mathcal{I}_{n}^{\mathrm{PQED}}=\frac{4}{\omega_{p, k}^{n}} \int_{0}^{\pi / 2} d \theta \frac{1}{\sqrt{1-x_{n} \sin ^{2}(\theta)}}=\frac{4}{\omega_{p, k}^{n}} K\left(x_{n}\right)$,

where $\omega_{p, k}^{n}$ and $x_{n}$ are given in Eqs. (60) and (61), respectively. The angular integral corresponds to an elliptical integral of the first kind, whereas the angular integral of the GN sector reads

$$
\mathcal{I}_{n}^{\mathrm{GN}}=\int_{0}^{2 \pi} d \theta \frac{1}{\frac{1}{g g_{0}}+\omega_{p, k}^{n} \sqrt{1-x_{n} \sin ^{2}(\theta)}}
$$

After some algebra, it is possible to write Eq. (B3) as the sum of three integrals, using the cosine double angle identity $\left(\cos (\theta)=1-2 \sin ^{2}(\theta / 2)\right)$, therefore, $\mathcal{I}_{n}^{\mathrm{GN}}$ is given by

$$
\begin{aligned}
\left(\mathcal{I}^{1}\right)_{n}^{\mathrm{GN}} & =\frac{2}{g g_{0} y_{n}} \int_{0}^{\pi} d \theta \frac{1}{1-y_{n} \sin ^{2}(\theta)}, \\
\left(\mathcal{I}^{2}\right)_{n}^{\mathrm{GN}} & =\frac{2}{\omega_{p, k}^{n}} \int_{0}^{\pi} d \theta \frac{1}{\sqrt{1-x_{n} \sin ^{2}(\theta)}}, \\
\left(\mathcal{I}^{3}\right)_{n}^{\mathrm{GN}} & =-\frac{2}{\omega_{p, k}^{n}} \int_{0}^{\pi} d \theta \frac{1}{\left(1-y_{n} \sin ^{2}(\theta)\right) \sqrt{1-x_{n} \sin ^{2}(\theta)}},
\end{aligned}
$$

where the integral $\left(\mathcal{I}^{1}\right)_{n}^{\mathrm{GN}}$ vanishes and, after properly writing the integrals $\left(\mathcal{I}^{2}\right)_{n}^{\mathrm{GN}}$ and $\left(\mathcal{I}^{3}\right)_{n}^{\mathrm{GN}}$, we obtain

$$
\mathcal{I}_{n}^{\mathrm{GN}}=\frac{4}{\omega_{p, k}^{n}}\left[K\left(x_{n}\right)-\frac{\Pi\left(y_{n}, x_{n}\right)}{1-g^{2} g_{0}^{2}\left(\omega_{p, k}^{n}\right)^{2}}\right],
$$

with $y_{n}$ being defined in Eq. (63). 
Finally, using these results, we find the mass function, namely,

$$
\begin{aligned}
\Sigma(p)= & \frac{C_{1} T}{(2 \pi)^{2}} \sum_{n=-\infty}^{\infty} \int_{0}^{\infty} d k 4 \frac{K\left(x_{n}\right)}{\omega_{p, k}^{n}} \frac{k \Sigma(k)}{(2 n+1)^{2} \pi^{2} T^{2}+k^{2}+\Sigma^{2}(k)} \\
& +\frac{C_{2} T}{(2 \pi)^{2}} \sum_{n=-\infty}^{\infty} \int_{0}^{\infty} d k \frac{4}{\omega_{p, k}^{n}}\left[K\left(x_{n}\right)-\frac{\Pi\left(y_{n}, x_{n}\right)}{1-g^{2} g_{0}^{2}\left(\omega_{p, k}^{n}\right)^{2}}\right] \\
& \times \frac{k \Sigma(k)}{(2 n+1)^{2} \pi^{2} T^{2}+k^{2}+\Sigma^{2}(k)} .
\end{aligned}
$$

\section{APPENDIX C: THE KERNEL EXPANSION}

The complete elliptic integral of the first and third kinds may be written in terms of hypergeometric function of two variables, for $\left|x_{n}\right|<1$ and $\left|y_{n}\right|<1$ [46], we have

$$
\begin{gathered}
K\left(x_{n}\right)=\frac{\pi}{2} F_{1}\left(\frac{1}{2} ; \frac{1}{2}, 1 ; 1, x_{n}, 0\right), \\
\Pi\left(y_{n}, x_{n}\right)=\frac{\pi}{2} F_{1}\left(\frac{1}{2} ; \frac{1}{2}, 1 ; 1, x_{n}, y_{n}\right) .
\end{gathered}
$$

The series of the Hypergeometric Appell F1 is given by

$F_{1}\left(\alpha ; \beta, \beta^{\prime}, \gamma ; x_{n}, y_{n}\right)=\sum_{l, q=0}^{\infty} \frac{(\alpha)_{l+q}(\beta)_{l}\left(\beta^{\prime}\right)_{q}}{(\gamma)_{l+q} l ! q !} x_{n}^{l} y_{n}^{q}$.

The standard procedure in the treatment of the SDEs is to consider two regions (infrared and ultraviolet) in the external momentum. After considering the zero-mode frequency $n=0$, we find

$2 \pi \frac{F_{1}\left(\frac{1}{2} ; \frac{1}{2}, 1 ; 1, x_{0}, 0\right)}{|p-k|}=\Theta(p-k) \frac{2 \pi}{p}+\Theta(k-p) \frac{2 \pi}{k}$

and

$$
\begin{aligned}
& \frac{2 \pi}{|p-k|}\left[F_{1}\left(\frac{1}{2} ; \frac{1}{2}, 1 ; 1, x_{0}, 0\right)-\frac{F_{1}\left(\frac{1}{2} ; \frac{1}{2}, 1 ; 1, x_{0}, y_{0}\right)}{1-g^{2} g_{0}^{2}\left(\omega_{p, k}^{n}\right)^{2}}\right] \\
& =\Theta(p-k) \frac{2 \pi}{p}+\Theta(k-p) \frac{2 \pi\left(g g_{0}\right)^{2} k}{\left[\left(g g_{0}\right)^{2} k^{2}-1\right]} .
\end{aligned}
$$

Therefore, the mass function reads

$$
\begin{aligned}
\Sigma(p)= & \frac{C_{1} T}{(2 \pi)^{2}} \int_{0}^{\infty} d k \frac{k \Sigma(k)}{\pi^{2} T^{2}+k^{2}+\Sigma^{2}(k)} \\
& \times\left[\Theta(p-k) \frac{2 \pi}{p}+\Theta(k-p) \frac{2 \pi}{k}\right] \\
& +\frac{C_{2} T}{(2 \pi)^{2}} \int_{0}^{\infty} d k \frac{k \Sigma(k)}{\pi^{2} T^{2}+k^{2}+\Sigma^{2}(k)} \\
& \times\left[\Theta(p-k) \frac{2 \pi}{p}+\Theta(k-p) \frac{2 \pi\left(g g_{0}\right)^{2} k}{\left[\left(g g_{0}\right)^{2} k^{2}-1\right]}\right],
\end{aligned}
$$

which is Eq. (67).

\section{APPENDIX D: THE EFFECTIVE POTENTIAL}

In this Appendix we show that the dynamically generated mass is energetically favorable in comparison to the symmetric phase (massless fermions).

In general grounds, one must use the solution of the Schwinger-Dyson equation into the Conrwall-JackiwTomboulis (CJT) potential [48] and find its minimum. This potential reads $V_{\text {CJT }} \propto-\ln Z$, where $Z$ is the partition function, and its quantum dynamics is given by a sum of 2-particle-irreducible (2PI) graphs, which depends on all of the full propagators and vertex interactions. Therefore, we may not calculate $V_{\mathrm{CJT}}$ for PQED, unless we assume a set of approximations. For $\mathrm{QED}_{3}$, within the large- $N$ limit, this has been calculated in Ref. [47], where it is shown that a dynamically generated mass $m \neq 0$ is the minimum of the CJT potential. Interesting, one could follow similar steps for PQED and find the same conclusion, because the only difference would be in the amplitude of the gauge-field propagator and this only changes the value of $m$ (and the critical parameters).

Next, we apply the bilocal-field representation proposed by Cahill-Roberts in Ref. [49] in order to provide an extra derivation of such result. For the sake of simplicity, we consider the rainbow-quenched PQED, which consists of neglecting quantum corrections to both the vertex interactions and the gauge-field propagator in Eq. (1). After using the bilocal-field representation for PQED, we find

$$
Z \approx \mathcal{N} e^{-\int d^{3}(x-y) \Gamma_{\mathrm{CR}}\left[B_{\mu}(x, y)\right]} \approx \mathcal{N} e^{-\Omega V_{\mathrm{CJT}}}
$$

where $B_{\mu}(x, y)$ is a bilocal-field satisfying the auxiliary condition $\left\langle B_{\mu}(x, y)\right\rangle=-e^{2}\left\langle\Delta(x-y) \bar{\psi}(x) \gamma_{\mu} \psi(y)\right\rangle, \mathcal{N}$ is a normalization constant, and $\Omega$ is the space-time volume. Furthermore, the Cahill-Roberts effective action reads

$$
\Gamma_{\mathrm{CR}}\left[B_{\mu}\right]=\frac{B^{\mu}(x, y) B_{\mu}(y, x)}{e^{2} \Delta(x-y)}-\operatorname{Tr} \ln \left[i \not \partial-\gamma^{\mu} B_{\mu}\right],
$$

where $\Delta(x-y)$ is the amplitude of the gauge-field propagator, obtained from $\Delta_{0, \mu \nu}(x-y)=\Delta(x-y) P_{\mu \nu}$ in Eq. (2). Note that $\gamma^{\mu} B_{\mu}=\Xi(x, y)$ is the electron self-energy, as defined in Eq. (16). From Eq. (D1), we conclude that the 
vacuum of the theory is given by the minimum of $V_{\mathrm{CJT}}$. Furthermore, note that Eq. (D2) is one term of the effective action derived in Ref. [50] for $\mathrm{QED}_{3}$ (see Appendix B).

From the Fourier transform of the motion equation $\delta \Gamma_{\mathrm{CR}}\left[B_{\mu}\right] / \delta B_{\mu}=0$, we find the standard SchwingerDyson equation for the electron self-energy $\Xi(p)$. Next, we consider a constant-field configuration within the rainbow-quenched approximation, i.e., we decompose the electron self-energy such that $\gamma^{\mu} B_{\mu}(p)=\Xi(p)=$ $(1-\bar{A}) \gamma^{\mu} p_{\mu}+\Sigma(p) \approx m$, where we have used that $\bar{A}=1$ (in order to neglect the renormalization of the electron wave function) and $\Sigma(p) \approx \Sigma(0)=m$, which represents the dynamically generated mass. Using this Schwinger-Dyson equation, for eliminating $\Delta(x-y)$ in Eq. (D2), we find

$V_{\mathrm{CJT}}[m]=\frac{1}{\pi^{2}} \int_{0}^{\infty} k^{2} d k\left[\frac{m^{2}}{k^{2}+m^{2}}-\ln \left(1+\frac{m^{2}}{k^{2}}\right)\right]$.

It turns out that $x /(1+x)-\ln (1+x)$ is a negative value for any positive $x \equiv m^{2} / k^{2}$, hence, the dynamical mass generation $m \neq 0$ is energetically favorable to $m=0$, as expected. It is worth to mention that Eq. (D3) also holds for $\mathrm{QED}_{3}$ (with a different $m$ ), as it has been shown in Eq. (4.9) of Ref. [47].
[1] K. S. Novoselov, A. K. Geim, S. V. Morozov, D. Jiang, Y. Zhang, S. V. Dubonos, I. V. Grigorieva, and A. A. Firsov, Electric field effect in atomically thin carbon films, Science 306, 666 (2004).

[2] A. H. Castro Neto, F. Guinea, N. M. R. Peres, K. S. Novoselov, and A. K. Geim, The electronic properties of graphene, Rev. Mod. Phys. 81, 109 (2009).

[3] G. Wang, A. Chernikov, M. M. Glazov, T. F. Heinz, X. Marie, T. Amand, and B. Urbaszek, Colloquium: Excitons in atomically thin transition metal dichalcogenides, Rev. Mod. Phys. 90, 021001 (2018).

[4] B. A. Bernevig with T. Hughes, Topological Insulators and Topological Superconductors (Princeton University Press, Princeton, NJ, 2013); S.-Q. Shen, Topological Insulators Dirac Equation in Condensed Matter, Spring Series in Solid-State Sciences (Springer, New York, 2012); M.Z. Hasan and C. L. Kane, Colloquium: Topological insulators, Rev. Mod. Phys. 82, 3045 (2010).

[5] C. L. Kane and E. J. Mele, Quantum Spin Hall Effect in Graphene, Phys. Rev. Lett. 95, 226801 (2005).

[6] F. D. M. Haldane, Model for a Quantum Hall Effect Without Landau Levels: Condensed-Matter Realization of the "Parity Anomaly", Phys. Rev. Lett. 61, 2015 (1988).

[7] E. C. Marino, L. O. Nascimento, V. S. Alves, and C. M. Smith, Interaction Induced Quantum Valley Hall Effect in Graphene, Phys. Rev. X 5, 011040 (2015).

[8] M. A. H. Vozmediano and F. Guinea, Effect of Coulomb interactions on the physical observables of graphene, Phys. Scr. T146, 014015 (2012); F. de Juan, A. G. Grushin, and M. A. H. Vozmediano, Renormalization of Coulomb interaction in graphene: Determining observable quantities, Phys. Rev. B 82, 125409 (2010).

[9] M. S. Foster and I. L. Aleiner, Grapehen via large N: A renormalization group study, Phys. Rev. B 77, 195413 (2008).

[10] J. González, F. Guinea, and M. A. H. Vozmediano, Marginal-Fermi-liquid behavior from two-dimensional Coulomb interaction, Phys. Rev. B 59, R2474 (1999).

[11] C. Popovici, C. S. Fischer, and L. von Smekal, Fermi velocity renormalization and dynamical gap generation in graphene, Phys. Rev. B 88, 205429 (2013).
[12] D. C. Elias, R. V. Gorbachev, A. S. Mayorov, S. V. Morozov, A. A. Zhukov, P. Blake, L. A. Ponomarenko, I. V. Grigorieva, K. S. Novoselov, F. Guinea, and A. K. Geim, Dirac cones reshaped by interaction effects in suspended graphene, Nat. Phys. 7, 701 (2011).

[13] L. Fernández, V. S. Alves, L. O. Nascimento, F. Peña, M. Gomes, and E. C. Marino, Renormalization of the band gap in 2D materials through the competition between electromagnetic and four-fermion interactions in large $\mathrm{N}$ expansion, Phys. Rev. D 102, 016020 (2020).

[14] E.C. Ahn, 2D materials for spintronic devices, NPJ 2D Mater. Appl. 4, 17 (2020).

[15] J. R. Schaibley, H. Yu, G. Clark, P. Rivera1, J. S. Ross, K. L. Seyler, W. Yao, and $\mathrm{X}$. $\mathrm{Xu}$, Valleytronics in 2D materials, Nat. Rev. Mater. 1, 16055 (2016).

[16] P. V. Nguyen, N. C. Teutsch, N. P. Wilson, J. Kahn, X. Xia, A. J. Graham, V. Kandyba, A. Giampietri, A. Barinov, G. C. Constantinescu, N. Yeung, N. D. M. Hine, X. Xu, D. H. Cobden, and N.R. Wilson, Visualizing electrostatic gating effects in two-dimensional heterostructures, Nature (London) 572, 220 (2019).

[17] F. Liu, M. E. Ziffer, K. R. Hansen, J. Wang, and X. Zhu, Direct Determination of Band-Gap Renormalization in the Photoexcited Monolayer $\mathrm{MoS}_{2}$, Phys. Rev. Lett. 122, 246803 (2019).

[18] P. Maris, Analytic structure of the full fermion propagator in quenched and unquenched QED, Phys. Rev. D 50, 4189 (1994).

[19] C. D. Roberts and A. G. Williams, Dyson-Schwinger equations and their application to hadronic physics, Prog. Part. Nucl. Phys. 33, 477 (1994); Dyson-Schwinger equations and their application to hadronic physics, last revised version arXiv:hep-ph/9403224.

[20] D. C. Curtis and M. R. Pennington, Truncating the Schwinger-Dyson equations: How multiplicative renormalizability and the ward identity restrict the three-point vertex in QED, Phys. Rev. D 42, 4165 (1990).

[21] T. Appelquist, M. J. Bowick, E. Cohler, and L. C. R. Wijewardhana, Chiral-Symmetry Breaking in $2+1$ Dimensions, Phys. Rev. Lett. 55, 1715 (1985). 
[22] I. F. Herbut, Interactions and Phase Transitions on Graphenes Honeycomb Lattice, Phys. Rev. Lett. 97, 146401 (2006).

[23] V. S. Alves, W. S. Elias, L. O. Nascimento, V. Juričić, and F. Peña, Chiral symmetry breaking in the pseudo-quantum electrodynamics, Phys. Rev. D 87, 125002 (2013).

[24] C.-Xu Zhang, G.-Zhu Liu, and M.-Qiu Huang, Dynamical fermion mass generation and exciton spectra in graphene, Phys. Rev. B 83, 115438 (2011).

[25] A. V. Kotikov and S. Teber, Critical behavior of reduced $Q E D_{4,3}$ and dynamical fermion gap generation in graphene, Phys. Rev. D 94, 114010 (2016).

[26] A. Bashir, C. Calcaneo-Roldan, L. X. Gutiérrez-Guerrero, and M. E. Tejeda-Yeomans, Critical number of flavors in QED, Phys. Rev. D 83, 033003 (2011).

[27] A. Bashir, A. Huet, and A. Raya, Gauge dependence of mass and condensate in chirally asymmetric phase of quenched three-dimensional QED, Phys. Rev. D 66, 025029 (2002).

[28] J. C. R. Bloch and M. R. Pennington, Can the photon quadratic divergence be canceled numerically? Mod. Phys. Lett. A 10, 1225 (1995).

[29] F. Akram, A. Bashir, L. X. Gutiérrez-Guerrero, B. Masud, J. Rodríguez-Quintero, C. Calcaneo-Roldan, and M.E. Tejeda-Yeomans, Vacuum polarization and dynamical chiral symmetry breaking: Phase diagram of QED with fourfermion contact interaction, Phys. Rev. D 87, 013011 (2013).

[30] G. W. Semenoff and L. C. R. Wijewardhana, Dynamical Mass Generation in 3D Four-Fermion Theory, Phys. Rev. Lett. 63, 2633 (1989).

[31] V. S. Alves, R. O. C. Junior, E. C. Marino, and L. O. Nascimento, Dynamical mass generation in pseudoquantum electrodynamics with four-fermion interactions, Phys. Rev. D 96, 034005 (2017).

[32] M. Gomes, R. S. Mendes, R. F. Ribeiro, and A. J. da Silva, Gauge structure, anomalies, and mass generation in a threedimensional Thirring model, Phys. Rev. D 43, 3516 (1991).

[33] B. A. Fayzullaev and E. Qayumov, Schwinger-Dyson type equations for some QFT models, arXiv:2006.04908.

[34] E. C. Marino, Quantum electrodynamics of particles on a plane and the Chern-Simons theory, Nucl. Phys. B408, 551 (1993).

[35] S. Teber, Electromagnetic current correlations in reduced quantum electrodynamics, Phys. Rev. D 86, 025005 (2012).
[36] L. O. Nascimento, V. S. Alves, F. Peña, C. M. Smith, and E. C. Marino, Chiral-symmetry breaking in pseudoquantum electrodynamics at finite temperature, Phys. Rev. D 92, 025018 (2015).

[37] D. Gross and A. Neveu, Dynamical symmetry breaking in asymptotically free field theories, Phys. Rev. D 10, 3235 (1974).

[38] A. Bender, C. D. Roberts, and L. V. Smekal, Goldstone theorem and diquark confinement beyond rainbow-ladder approximation, Phys. Lett. B 380, 7 (1996).

[39] J.S. Ball and T.-W. Chiu, Analytic properties of the vertex function in gauge theories. II, Phys. Rev. D 22, 2550 (1980).

[40] K. Kondo, Y. Kikukawa, and H. Mino, Phase structure of quantum electrodynamics in the framework of the Schwinger-Dyson equation, Phys. Lett. B 220, 270 (1989).

[41] J. I. Kapusta, Finite-Temperature Field Theory (Cambridge University Press, Cambridge, England, 1989).

[42] V. S. Alves, L. Fernández, S. Lepe, L. O. Nascimento, and F. Peña, Renormalization group approach in a Lifshitz-like Gross-Neveu-Thirring model, Phys. Rev. D 97, 065015 (2018).

[43] G. Arfken, Mathematical Methods for Physicists, 3rd ed. (Academic Press, New York, 1985).

[44] I. J. R. Aitchison, Beyond the instantaneous-exchange approximation for three-dimensional QED at finite temperature, Z. Phys. C 67, 303 (1995).

[45] A. Ayala and A. Bashir, Dynamical mass generation fourfermions in quenched quantum electrodynamics at finite temperature, Phys. Rev. D 67, 076005 (2003).

[46] F. W. J. Olver, D. W. Lozier, R. F. Boisvert, and Charles W. Clark, NIST Handbook of Mathematical Functions, 1st ed. (NIST and Cambridge University Press, New York, 2010).

[47] T. W. Appelquist, M. Bowick, D. Karabali, and L. C. R. Wijewardhana, Spontaneous chiral-symmetry breaking in three-dimensional QED, Phys. Rev. D 33, 3704 (1986).

[48] J. M. Cornwall, R. Jackiw, and E. Tomboulis, Effective action for composite operators, Phys. Rev. D 10, 2428 (1974).

[49] R. T. Cahill and C.D. Roberts, Soliton bag models of hadrons from QCD, Phys. Rev. D 32, 2419 (1985).

[50] C. J. Burden, Bound states from the Bethe-Salpeter equation in QED3, Nucl. Phys. 387, 419 (1992). 